\title{
Formación de habilidades investigativas: un reto para la Educación Superior Universitaria Salvadoreña
}

\section{Research Skills Training: a Challenger for Salvadoran Higher Education}

\author{
Lourdes Elizabeth Prudencio Coreas \\ Candidata a doctora en Educación, con \\ especialidad en Educación Superior, Universidad de \\ El Salvador \\ lourdes.prudencio@ues.edu.sv \\ https://orcid.org/0000-0003-2915-345X
}

Recibido 18/10/2021

Aceptado 16/11/2021

\section{Referencia}

Prudencio Coreas, L. E. (2021). Formación de habilidades investigativas: un reto para la Educación Superior Universitaria Salvadoreña. Revista Guatemalteca de Educación Superior, 5(1), 88101. https://doi.org/10.46954/revistages.v5i1.77

\section{Resumen}

OBJETIVO: sistematizar los fundamentos teóricos de la formación de habilidades investigativas en la educación superior universitaria. MÉTODO: el método para su elaboración fue el método de análisis documental, realizando una sistematización de la literatura científica relacionada con el área de conocimiento de la formación de habilidades investigativas, de autores internacionales que presentan sus diferentes posiciones teóricas, particularmente en América Latina. RESULTADOS: el resultado científico de mayor relevancia es concebir la formación y desarrollo de habilidades investigativas como un proceso dialéctico, largo 
y complejo que involucra a cada disciplina y asignatura de las diferentes carreras que se imparten en la educación superior universitaria. Además, se constata la existencia de una dispersión de términos de la asunción de un sistema categorial referido al proceso de formación de las habilidades investigativas. CONCLUSIÓN: según las tendencias mundiales de la educación superior, el énfasis en la educación universitaria debe centrarse en la complementariedad de la formación académica de orden conceptual teórica con el conocimiento técnico práctico, lo que implica que el estudiante, además de conocer tiene que saber hacer, en donde la investigación tiene un rol importante en el proceso de aprendizaje.

\section{Abstract}

OBJECTIVE: itis the theoretical foundations of the formation of research skills in university higher education. METHOD: the method for its elaboration was the method of documentary analysis, carrying out a systematization of the scientific literature related to the area of knowledge of the formation of investigative skills, of international authors who present their different theoretical positions, particularly in Latin America. RESULTS: the most relevant scientific result is to conceive the training and development of research skills as a dialectical, long, and complex process that involves each discipline and subject of the different careers taught in university higher education. In addition, the existence of a dispersion of terms of the assumption of a categorial system referring to the process of formation of research skills is observed. CONCLUSION: according to the global trends of higher education, the emphasis on university education should focus on the complementarity of academic training of theoretical conceptual order with practical technical knowledge, which implies that the student, in addition to knowing has to know how to do, where research has an important role in the learning process.
Palabras clave:

educación superior, investigación, formación y habilidades investigativas.
Keywords:

Higher education, research, training, and research skills. 


\section{Introducción}

La Formación de habilidades investigativas: Un reto para la Educación Superior Universitaria Salvadoreña, es un tema de relevancia académica-científica en el contexto de la sociedad del conocimiento. Los retos de la sociedad actual demandan de la educación superior universitaria la necesidad de potenciar la investigación científica y la formación para la misma, esta necesidad debe ser objeto de reflexión para la comunidad académica.

La investigación como una práctica sociohistórica, ha sido a lo largo de la historia de la sociedad, una necesidad de los seres humanos para conocer, explicar y transformar sus entornos socioambientales. Esta necesidad ha llevado a la sociedad moderna a crear y desarrollar diferentes procesos y prácticas sociales a fin de resolver los problemas de los grupos, comunidades e instituciones sociales, constituyéndose en la principal vía para la conservación, reproducción y desarrollo de la cultura y todo lo que en ella se incluye.

Situaciones tan complejas, como las provocadas por la Pandemia por COVID-19, han demostrado una vez más la necesidad de la participación de todos los actores, desde la ciencia y la solidaridad para salvar la humanidad, situación que trae de nuevo al debate académico, el papel de las universidades en la formación de una cultura científica y de promover en estrecha relación con la sociedad, el desarrollo de la ciencia, tecnología e innovación. Sin embargo, los aportes científicos en la actualidad generalmente provienen de países desarrollados.

Los factores que inciden en el bajo desarrollo de la ciencia a nivel social y educacional en los países en vías de desarrollo, como es el caso salvadoreño, son varios, entre los que se destacan, el poco apoyo financiero del Estado y la carencia de una cultura investigativa en las Instituciones de Educación Superior (IES). Estos factores se manifiestan en los países de América Latina, con mayor acento en países del Caribe y los centroamericanos, como El Salvador, donde se concentran los indicadores más bajos de producción científica. 
Aunque la problemática anterior es multicausal, el cambio puede comenzar a partir de asumir un modelo formativo, donde la investigación y la formación investigativa, sean parte esencial de las funciones de las IES, por ello, las universidades del siglo XXI deben incorporar la investigación como práctica, y no como una declaración formal en sus documentos oficiales.

En tal sentido, a criterio de la autora, las universidades salvadoreñas deberán hacer de la investigación, la principal estrategia para impulsar el desarrollo y atender las necesidades de la sociedad. De ahí que, el objetivo de este ensayo es convocar a la reflexión crítica para abordar uno de los problemas actuales de la educación superior salvadoreña, como es: el desarrollo de las habilidades investigativas como parte sustancial del proceso de formación de los profesionales en el nivel superior universitario. Para ello es necesario abordar la problemática bajo dos aristas: desde la perspectiva teórica-reflexiva y desde la realidad de la educación superior salvadoreña.

El trabajo es el resultado de la sistematización teórica realizada en la literatura especializada, así como de la aplicación del análisis documental como método científico, realizado para fundamentar el capítulo uno de la tesis doctoral de la autora.

\section{Contenido}

\section{El contexto de la Educación Superior}

En la Conferencia Mundial de la Educación Superior celebrada en Paris en el 2009, se establece que, ante la complejidad de los desafíos mundiales, presentes y futuros, la educación superior tiene la responsabilidad social de hacer avanzar la comprensión de problemas polifacéticos con dimensiones sociales, económicas, científicas y culturales, así como la capacidad de hacerles frente. Se establece que la educación superior debería asumir el liderazgo social en materia de creación de conocimientos de alcance mundial para abordar retos mundiales (Organización de las Naciones Unidas para la Educación, la Ciencia y la Tecnología [UNESCO] 2009). 
La conferencia anterior demostró el compromiso de todos los actores clave de reconocer la educación superior como un bien público, y como la principal fuerza para construir una sociedad del conocimiento diversa e inclusiva y fomentar la investigación, la innovación y la creatividad, aspiraciones que aún siguen teniendo una vigencia sustantiva, por lo que se debe continuar trabajando para lograr dichos propósitos.

Las situaciones y problemáticas que enfrenta la sociedad actual como: Crisis económica, situaciones ambientales extremas, pandemia, entre otras, reclaman la urgente necesidad de contar con ciudadanos socialmente comprometidos y responsables, que sean capaces de contribuir a los cambios que demandan los tiempos actuales. Esta necesidad se traslada a las Instituciones de Educación Superior, particularmente las universidades, las que, como parte de su misión social y función central, están comprometidas a orientar la formación de los profesionales, tomando en cuenta las exigencias de la sociedad, con sensibilidad social y una preparación técnica y científica para el servicio de esta.

Sin duda, el desafío anterior, no será posible enfrentarlo, si estas instituciones no fortalecen o refuerzan la cooperación con los sectores sociales y económicos, analizando y previendo las necesidades y problemáticas de sus entornos y desarrollando investigaciones que ayuden a explicar, entender y transformar la realidad circundante.

En países en desarrollo, como El Salvador, la formación universitaria está llamada a vincularse al contexto social, indagar en problemáticas interconectadas, mismas que necesitan soluciones creativas basadas en la investigación y la innovación. Por ello, las universidades deben habituarse al complejo trabajo académico de investigación, porque en el ejercicio de las profesiones y para ser competentes, los profesionales formados, deben mantenerse actualizados a través de una cultura científicoinvestigativa. 
De ahí que, trabajar el componente investigativo constituye un punto de inflexión para alcanzar una universidad pertinente, donde se trascienda las prácticas y actuaciones tradicionales de la docencia y extensión universitaria, hacia una relación de colaboración y articulación entre ellas, capaz de dar respuesta a los retos sociales de la realidad actual.

Por ello, la educación superior juega un rol estratégico de vital importancia, tanto en la generación de conocimientos e innovaciones tecnológicas, como en la formación de profesionales capaces de estudiar y aportar a la comprensión y solución de los problemas del entorno.

Sin duda, entre la academia y la sociedad debe establecerse un diálogo que produzca resultados tangibles y pertinentes; por ejemplo: proveer profesionales capaces de responder a las necesidades sociales, desarrollar investigaciones oportunas sobre los problemas sociales, económicos y ambientales, explicar y proponer soluciones a los mismos, es decir trascender de la simple generación del conocimiento a la difusión y transferencia que contribuya al bienestar social.

La generación del conocimiento mediante la investigación científica involucra tanto a docentes como estudiantes durante el proceso de formación académica-científica. De ahí, que la investigación debe ser un componente esencial del proceso de formación de los estudiantes, de tal forma, que los prepare científicamente para su futuro desempeño laboral y profesional en la sociedad, especialmente ahora que se vive en una sociedad en la que el conocimiento es el principal motor de desarrollo económico y social.

Esta preparación científica, implica incorporar el componente investigativo desde los primeros años de su formación, que les permita desarrollar capacidades y habilidades investigativas, las que se logran a través de un proceso de formación en el que los estudiantes aprenden desarrollando acciones o actividades de investigación. 
De acuerdo con Machado y Montes (2009) la formación científica en el ámbito de la educación superior del nuevo milenio debe centrarse en dos aspectos esenciales: 1. Preparar al futuro profesional para asimilar creadoramente los conocimientos científicos y tecnológicos que forman parte del mundo actual y transformar la realidad a través de la investigación. 2. Formar en él la actitud positiva y con valores propios de la cultura científica, esto es, una disposición indagadora, base de la investigación en su sentido más amplio, en cualquier esfera del conocimiento humano.

De acuerdo con las tendencias mundiales de la educación superior, el énfasis de la educación universitaria está centrado en la complementariedad de la formación académica universitaria de orden conceptual teórica con el conocimiento técnico práctico, lo que implica que el estudiante, además de conocer tiene que saber hacer, en donde la investigación tiene un rol importante en el proceso de aprendizaje.

\section{La formación y desarrollo de las habilidades investigativas}

El abordaje de la formación investigativa demanda ciertas precisiones conceptuales y relaciones que son necesarias para entender la lógica de aplicación de esta concepción en un currículo universitario. Una de las premisas conceptuales a puntualizar es la relación entre la formación y el desarrollo, como dos procesos básicos que se manifiestan en la formación de habilidades investigativas.

El concepto de formación como se sabe tiene un carácter polisémico, atribuido en algunos casos al de educación, en otras al de instrucción, entrenamiento y capacitación. También, se le acompaña de adjetivos como: "formación continua, formación permanente, formación en ejercicio y de perfeccionamiento" (Moreno, 1997, p. 39). 
Otros autores entienden la formación desde una base cultural y antropológica, concibiéndola como un proceso permanente, no restringido a un espacio institucional o académico, sino más bien como un proceso que se desarrolla durante toda la vida, en todos los espacios donde se lleva a cabo la interacción humana.

Por otra parte, en el campo de la pedagogía, la formación como categoría, es entendida como: "Un acto intencional concreto en el que las subjetividades de quién educa o forma y de quién es educado o formado se encuentran con el fin de producir un salto cualitativo en la construcción del conocimiento" (Toro et al., 2015, p. 140). La formación entendida como categoría pedagógica forma sujetos activos y protagonistas de su propio proceso.

Otro concepto vinculado al de "formación" es el de "desarrollo", ambos constituyen "etapas dialécticamente relacionadas, donde la primera representa la orientación adecuada sobre los modos de actuación y la segunda implica la ejercitación de estos" (Martínez y Márquez, 2014, p. 35). De ahí que, formación y desarrollo constituyen un binomio dialéctico que se manifiesta con sus particularidades en la formación investigativa, como función de la educación superior, donde el concepto de formación lleva inexorablemente al de desarrollo, es decir, para lograr el desarrollo de las habilidades hay que formarlas en el proceso de enseñanza aprendizaje de los estudiantes.

En cuanto al análisis de la formación investigativa en el nivel superior universitario, existen diferentes perspectivas o lógicas. Al respecto, se revisó un estudio de Moreno (2005), en el que se identifican dos lógicas o racionalidades que han prevalecido en los procesos de formación investigativa de la educación superior latinoamericana. Una de ellas es la lógica "de incorporar contenidos y de pasos metodológicos", para la formación investigativa; la segunda, se refiere a la lógica del "desarrollo de habilidades", la que ha sido la predominante en lo que va de este siglo en los espacios curriculares de la educación superior. 
Bajo la primera lógica se crean y se ofrecen en las universidades, cursos de metodología con la intención de formar para la investigación, siendo la de mayor divulgación durante las últimas décadas del siglo XX. La segunda, está orientada al desarrollo de habilidades. Esta lógica cobra relevancia en la última década del siglo XX, donde se percibe el paso de una lógica de dominio de ciertos contenidos a una lógica de desarrollo de habilidades; de una concepción de pasos a seguir a una de procesos y operaciones que se dan en la generación de conocimientos; pero también se empieza a percibir la aparición de ciertos rasgos de conducta o actitudes que habrán de cultivarse en el investigador en formación, quizá como expresión de una mayor conciencia de que es la totalidad de la persona la que se compromete en una práctica como la de la investigación (Moreno,2005).

Bajo esta segunda lógica, los estudios de Moreno (2005), parten del supuesto "que la formación para la investigación implica aprendizajes en el campo de los conocimientos, las habilidades, los hábitos, las actitudes y los valores, pero el núcleo fundamental e integrador de dichos aprendizajes es el desarrollo de habilidades investigativas" (p. 227).

Partiendo de la lógica de desarrollo de habilidades, la formación investigativa implica un proceso continuo donde se desarrollan habilidades, capacidades y valores de la actividad científica.

El concepto anterior establece que las universidades tanto en pregrado como en posgrado deben fortalecer la formación de habilidades investigativas, de una manera continua, pero con propósitos diferentes, según el nivel que se trate. Para el pregrado se busca trabajar y fortalecer las competencias científico-investigativas básicas. Si bien es cierto, que la mayoría de los profesionales formados en las Instituciones de Educación Superior universitarias no serán investigadores de profesión, estos profesionales deben tener la capacidad de hacer investigación y realizarla cuando sea necesaria en su campo profesional-laboral. 
Es importante destacar que, en el nivel de pregrado, se refieren habilidades investigativas profesionales comunes a cualquier carrera universitaria, siempre y cuando estas impliquen el dominio de las acciones prácticas que a partir de los conocimientos adquiridos puedan ir a la búsqueda y solución de los problemas, aplicando los procedimientos, herramientas e instrumentos del método científico.

La formación y desarrollo de habilidades investigativas debe entenderse como un proceso dialéctico, largo y complejo que implica a cada disciplina, asignatura o componente de las diferentes carreras universitarias, con una perspectiva inter, multi y transdisciplinaria para el logro de su dominio en los estudiantes, constituyéndose en el elemento esencial de la dirección del proceso enseñanza-aprendizaje.

De ahí la necesidad de incorporar las habilidades investigativas como habilidades profesionales inherentes a todas las disciplinas, asignaturas y componentes del currículo de las carreras que se cursan en la educación superior" (Machado y Montes de Oca, 2009, p. 22). Estas habilidades permiten la ejecución de los conocimientos teóricos acerca de la metodología de la investigación científica en la práctica investigativa, utilizando para ello técnicas, métodos, estrategias y metodologías para la realización de una investigación científica.

Entendido el concepto de habilidades investigativas como "el dominio de la acción que se despliega para solucionar tareas investigativas en el ámbito docente, laboral y propiamente investigativo con los recursos de la metodología de la ciencia" (Machado et al., 2008 p.164), se proponen diferentes estrategias o acciones enfocadas a la formación y desarrollo de estas habilidades en el nivel superior universitario. Dentro de estas se encuentra: el trabajo práctico orientado a través de la tarea docente, la que se define como "la célula del proceso formativo donde, bajo la dirección y orientación del profesor, el estudiante ejecuta diversas acciones, utilizando la lógica y la metodología de la ciencia, tendientes a la solución de situaciones y problemas que acontecen" (Machado et al., 2008, p.164). 
Otros autores como Casanova et al., (2020), comparten al igual que Machado et al., (2008), que la resolución de tarea docente o investigativa es la acción fundamental para formar habilidades investigativas en los estudiantes de pregrado. Para estos autores, la tarea docente es la célula de la actividad conjunta profesor-estudiante, donde se diseñan las actividades que el estudiante debe realizar en clase y fuera de ésta, vinculadas a la búsqueda y adquisición del contenido de enseñanza que incluye los conocimientos, las habilidades y los valores a formar en los estudiantes.

La tarea docente se ha convertido en una tendencia y alternativa pedagógica en América Latina para la formación y desarrollo de las habilidades investigativas. Sin embargo, para lograrlo es necesario que los profesores planifiquen previamente este tipo de tareas contextualizadas o ajustadas a cada una de las profesiones, combinando acciones y operaciones a desarrollar en el proceso enseñanza- aprendizaje.

Una experiencia exitosa para fortalecer la formación y las habilidades investigativas ha sido el Programa Institucional de Semilleros de Investigación, implementada en la Universidad Católica de Colombia. Estos semilleros agrupan diferentes actividades, por lo general desarrollan actividades colectivas como: seminarios de investigación, talleres, foros, mesas redondas, paneles, charlas y sesiones de discusión. Así también se realizan trabajos individuales como lectura y análisis de documentos, generación de escritos, diseño de esquemas y prototipos, formulación de proyectos entre otros (Guerrero, 2007). Esta es una estrategia, que, de acuerdo con las investigaciones consultadas, ha producido resultados positivos en las instituciones donde ha sido aplicada, por lo que podría convertirse en una estrategia a considerar para formar y desarrollar las habilidades investigativas en el nivel superior universitario. 


\section{Conclusión}

Según las tendencias mundiales de la educación superior, la educación universitaria debe centrarse en la complementariedad de la formación conceptual-teórica con el conocimiento teóricopráctico, lo que significa que el estudiante, además de conocer, debe saber hacer, es decir aplicar los conocimientos adquiridos en situaciones concretas de su realidad. De ahí que, la formación investigativa es un proceso intencionado, que involucra en el ámbito universitario la docencia y la extensión social, permitiendo con ello el desarrollo de conocimientos, habilidades, capacidades y valores, base de una cultura científica que se construye desde los diferentes espacios curriculares.

La transformación del proceso de enseñanza -aprendizaje para la formación investigativa, requiere de un debate previo dentro de la comunidad universitaria, que permita establecer los lineamientos teóricos y metodológicos a sumir para lograr el vínculo pertinente de la universidad con el contexto y la formación integral de sus egresados. La escasez de formación del potencial humano, y la ausencia de políticas educativas coherentes que contribuyan a formar profesionales capaces de enfrentar los nuevos desafíos, deben ser retomados como problemáticas a resolver en el corto y mediano plazo.

Asumir la formación investigativa implica diseñar acciones orientadas a la asimilación y desarrollo de los conocimientos, habilidades y actitudes necesarias para que los estudiantes puedan desempeñar con eficacia y eficiencia las actividades de su práctica profesional, vinculadas con la investigación científica, el desarrollo tecnológico y la innovación independientemente de la estrategia a implementar, lo que se persigue con la formación de habilidades investigativas, como parte esencial del proceso de formación científica del estudiante de pregrado, es que se permita a los futuros profesionales identificar y dar solución a problemas científicos y asumir con responsabilidad la misión de transformar el entorno socioeconómico y cultural en el que desarrollan su práctica profesional. 


\section{Referencias bibliográficas}

Casanova, T. A., González, C. Y., Vásquez, M. G., y Asqui, J. E (2020). Acciones Para Formar Habilidades Investigativas en Estudiantes De La Carrera De Educación Infantil en El Contexto Ecuatoriano. Pedagogía Universitaria, 24(3), 100-119. http://www.dspace.uce.edu.ec/ bitstream/25000/20809/1/T-UCE-0010-FIL-792.pdf

Guerrero-Euseda, M. (2007). Formación para la Investigación desde el pregrado. Universidad Católica de Colombia. Acta Colombiana de Psicología 10(2): 190-192. http:// www.scielo.org.co/pdf/acp/v10n2/v10n2a18.pdf

Machado, E. F., Montes de Oca, N y Mena, A (2008). El Desarrollo de Habilidades Investigativas como objetivo educativo en las condiciones de la universalización de La Educación Superior. Pedagogía Universitaria, XIII(1), 156-180. http://revistas.mes.edu.cu/PedagogiaUniversitaria/articulos/2008/numero/189408108.pdf/ viewUniversidadde Antioquia, Facultad de Educación, Lógica y teoría de conjuntos, [2006].

Machado, E. F, y Montes de Oca R. N (2009). Las habilidades investigativas y la nueva Universidad: Terminus a quo a la polémica y la discusión. Humanidades Médicas, 9(1) http://scielo.sld.cu/scielo.php?script=sci_ arttext\&pid=S1727-81202009000100002\&lng=es\&tlng =es.

Martínez, D. y Márquez, D. L.(2014). Tendencias de la formación y desarrollo de habilidades investigativas en el pregrado. TLATEMOANI, (17), 35-46. https://dialnet. unirioja.es/servlet/articulo?codigo $=7350895$

Moreno, M.G. (2005). Potenciar la educación. Un currículum transversal de formación para la investigación. Revista Electrónica Iberoamericana sobre Calidad, Eficacia y Cambio en Educación, 3 (1), 520-540. http://dialnet. unirioja.es/servlet/articulo?codigo $=1130331$ 
Toro, I. D., Saldarriaga , J. G., León , M., Martínez, J., y Arias, O. (2015). Competencias Docentes para la Enseñanza de la Metodología de la investigación y la Evaluación de trabajo de grado y tesis doctorales en Admistracion. AGU. USB Medellin-Colombia , 15(1), 137-151. http://www. scielo.org.co/scielo.php?script=sci_arttext\&pid=S1657$80312015000100008 \&$ lng $=$ en\&tlng=es.

UNESCO (2009). Conferencia Mundial sobre la Educación Superior: La Nueva Dinámica de la Educación Superior y la Investigación para el Cambio Social y el Desarrollo. http:// www.unesco.org/education/WCHE2009/comunicado_ es.pdf.

\section{Sobre la autora}

Lourdes Elizabeth Prudencio Coreas, es profesora universitaria de la Facultad Multidisciplinaria Oriental, Universidad de El Salvador. Graduada de Licenciada en Sociología de la Universidad de El Salvador, cuanta con una Maestría en Métodos y Técnicas de Investigación Social. Actualmente es candidata a doctora en Educación con especialidad en Educación Superior, Convenio Universidades de La Habana y Ciencias Pedagógicas de Cuba y Universidad de El Salvador.

\section{Conflicto de intereses}

Declara no tener ningún conflicto de intereses.

\section{Declaración de consentimiento informado}

El estudio se realizó con fines educativos, respetando el Código de ética y buenas prácticas editoriales de publicación.

\footnotetext{
Derechos de uso

Copyright@ 2021 por Lourdes Elizabeth Prudencio Coreas

(c) (1)

Este texto está protegido por la Licencia Creative Commons Atribución 4.0 Internacional.

Usted es libre para compartir, copiar y redistribuir el material en cualquier medio o formato y adaptar el documento, remezclar, transformar y crear a partir del material para cualquier propósito, incluso comercialmente, siempre que cumpla la condición de atribución: usted debe reconocer el crédito de una obra de manera adecuada, proporcionar un enlace a la licencia, e indicar si se han realizado cambios. Puede hacerlo en cualquier forma razonable, pero no de forma tal que sugiera que tiene el apoyo del licenciante o lo recibe por el uso que hace.
} 